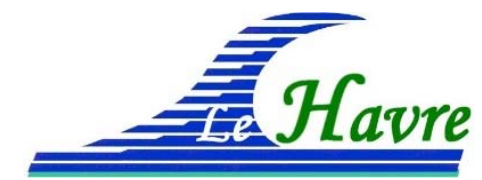

XVI èmes Journées Nationales Génie Côtier - Génie Civil

Le Havre, 2020

DOI:10.5150/jngcgc.2020.094 (C) Editions Paralia CFL

disponible en ligne - http://www.paralia.fr - available online

\title{
Perspectives des ressources minérales offshores du bassin côtier congolais (Congo-Brazzaville)
}

\author{
Yvon BAKADILA-MONA ${ }^{1}$, Hypolite ODIKA ${ }^{1}$, David BILEMBI ${ }^{1}$
}

1. Université Marien Ngouabi, Ecole Nationale Supérieure Polytechnique, B.P 69, Brazzaville, Congo.

bak.yvon@post.cz; hypoliteodika133@gmail.com

\section{Résumé :}

L'existence des indices d'hydrocarbures du bassin côtier congolais, était connue depuis longtemps par les premières prospections, menées dès 1928 jusqu'en 1940. Aujourd'hui, l'essentiel de la connaissance du bassin côtier, provient cependant des études de subsurface, et cela à partir des sondages de recherche pétrolière, qui sont appuyées par de nombreuses études géophysiques.

L'offshore du bassin côtier congolais est doté des ressources minérales importantes et d'un grand potentiel géologique confirmé par les prospections réalisées par les entreprises pétrolières étrangères depuis 1968. Au cours des années 1970, le développement du secteur offshore est devenu l'axe prioritaire de l'économie congolaise. La hausse production pétrolière des années 2010, essentiellement par la mise en production du champ de "MOHO NORD" en 2017, a permis au Congo d'occuper la troisième place des pays producteurs du pétrole en Afrique subsaharienne après le Nigeria et l'Angola, puis encore à l'adhésion du Congo à l'OPEP (organisation des pays exportateurs du pétrole). La production pétrolière augure donc des bonnes perspectives pour le pays, dont l'économie dépend essentiellement des ressources du secteur pétrolier.

Mots clés: Ressources minérales, Hydrocarbures, Pétrole, Gaz naturel, Gisement, Champs, Réserves, Offshore, On-shore, Bassin côtier, Congo-Brazzaville. 


\section{Hors session}

\section{Introduction}

La République du Congo (Congo-Brazzaville), est située en Afrique centrale. Elle chevauche la ligne équatoriale et couvre une superficie de $342000 \mathrm{~km}^{2}$ avec une population d'environ quatre (4) millions d'habitants. Le pays est limité :

- au nord par la République centrafricaine et la République du Cameroun ;

- à l'est par la République Démocratique du Congo ;

- au sud par la République d'Angola (Cabinda) ;

- et à l'ouest par la République du Gabon.

La République du Congo a un littoral maritime de $170 \mathrm{~km}$ le long de l'océan Atlantique. En République du Congo, environ deux tiers du PIB national et $80 \%$ des exportations sont reliés au pétrole. La performance économique du pays de ces dernières décennies résulte de cette richesse nationale exploitée avec succès depuis 40 ans. Avec les réserves existantes, les découvertes récentes, les investissements prévus, et si les cours du baril auront des tendances favorables, l'après-pétrole se situe probablement dans un horizon encore lointain.

Malgré les importantes réserves et une production journalière autour de 280000 barils, 95\% du pétrole brut produit est exporté, alors que le pays couvre $41 \%$ de ses besoins par les importations. Les produits pétroliers représentent à peine $7 \%$ de la demande en énergie. Ils sont essentiellement composés des carburants (essence, gasoil et fuel pour les transports maritimes, fluviales, routiers et aériens) et le pétrole lampant pour l'éclairage et la cuisson des ménages. Les réserves pétrolières du Congo sont estimées à 1,6 milliards de barils.

Le bassin côtier congolais (voir figure 1) représente $43300 \mathrm{~km}^{2}$ en offshore et 11900 $\mathrm{km}^{2}$ en on-shore, environ 2 milliards de barils possibles et 500 millions à prouver dans les champs en exploitation et 858 millions de barils de réserves possibles à prouver dans les titres de recherche. 


\section{BASSIN COTIER}

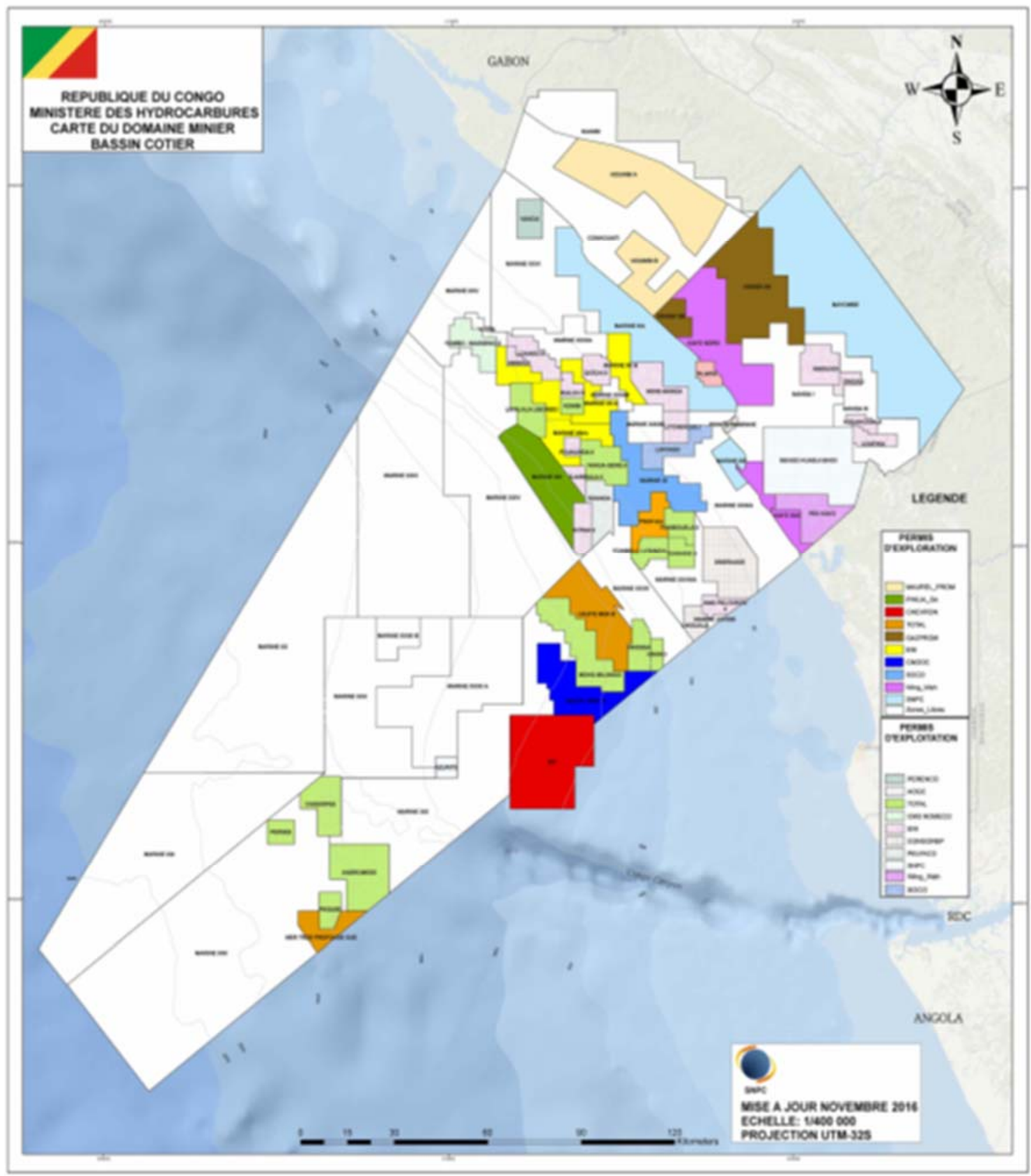

Figure 1. Bassin côtier congolais, (source SNPC, 2017a).

\section{Ressources minérales offshores}

Dans le bassin côtier Congolais, les ressources minérales offshores (pétrole et gaz) sont liées aux formations Albiennes et Miocènes, mais aussi aux formations sous-explorées de l'anté-salifères (voir figures 2 et 3). 


\section{Hors session}

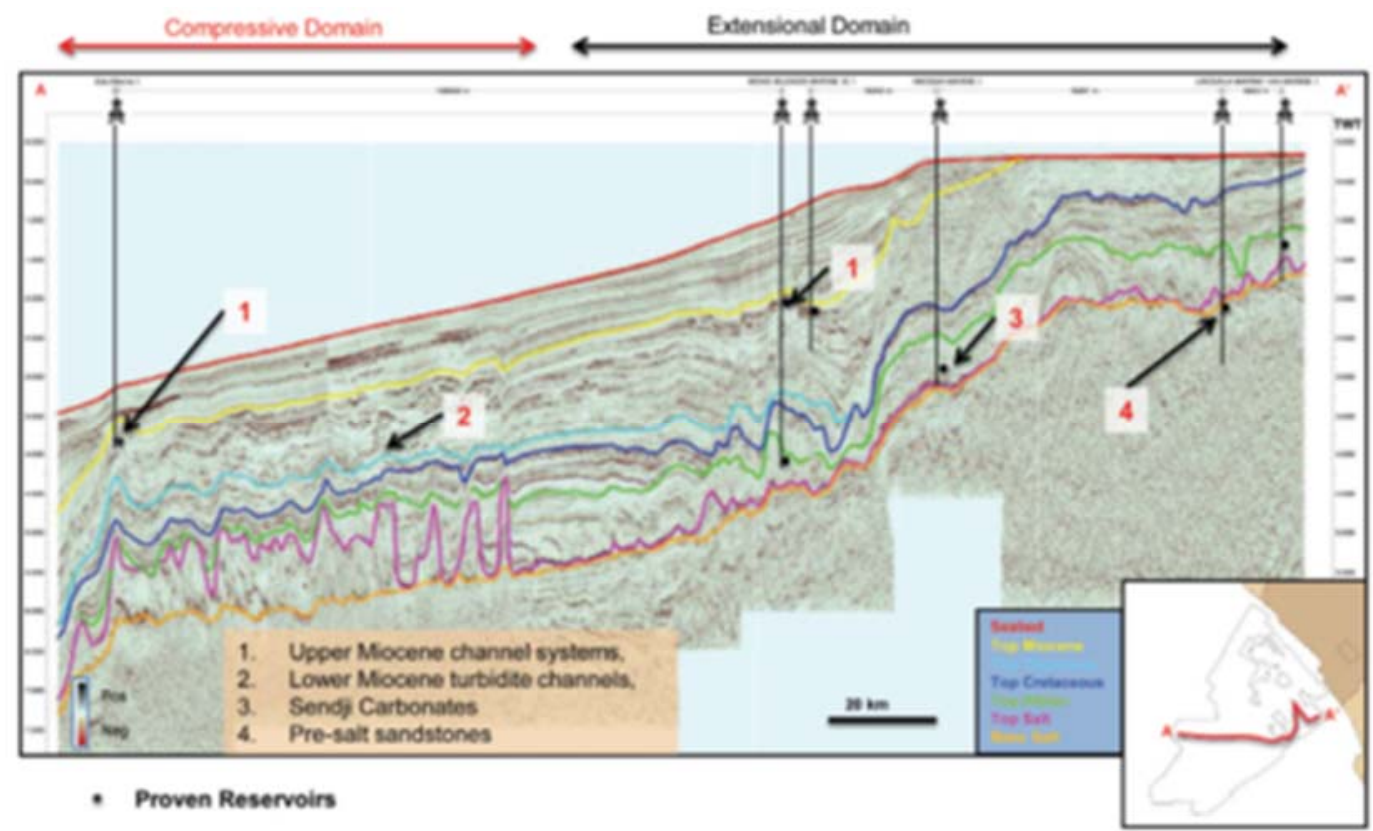

Figure 2. Coupe latérale de l'offshore du bassin côtier Congolais, (source SNPC, 2017b).

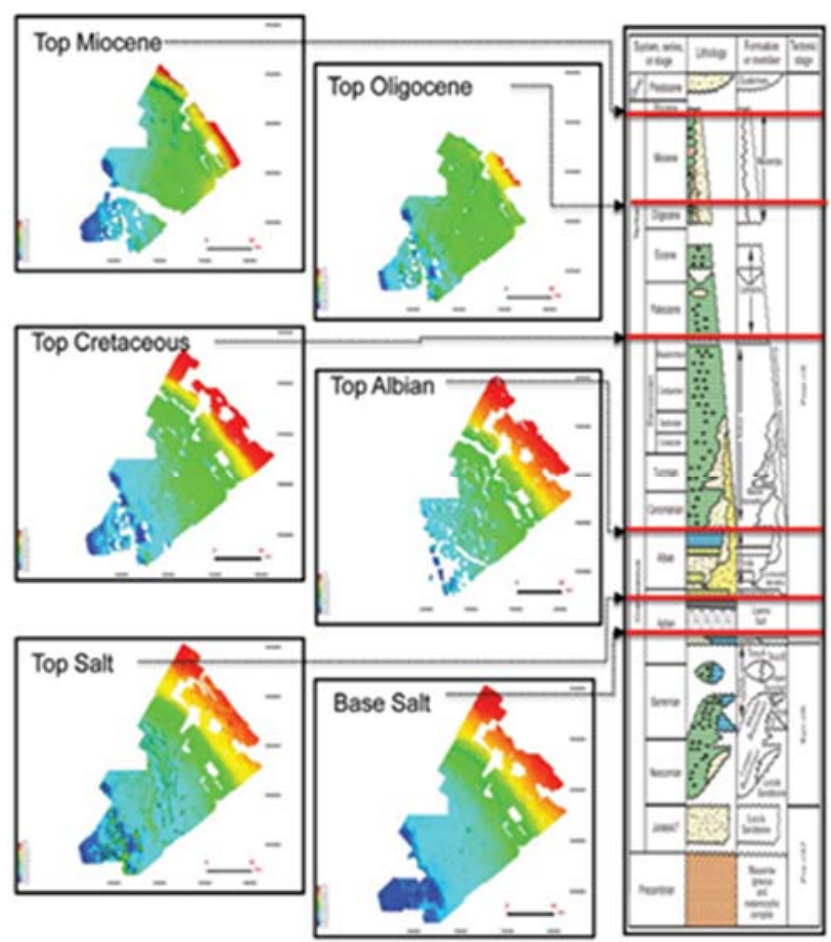

Figure 3. Coupe de la stratification du bassin en relation avec les accumulations des hydrocarbures, (source SNPC, 2017b). 


\section{XVI'mes Journées Nationales Génie Côtier - Génie Civil \\ Le Havre 2020}

\subsection{Le pétrole}

Le premier inventaire des hydrocarbures au Congo a été réalisé entre 1928 et 1940. Au début des années 30, les indices superficiels bitumineux décelés sur les marges du bassin et à Pointe-Noire (d'où son nom) attirent l'attention des géologues pétroliers.

Les premières études ont étés effectuées par LEBEDEFF \& SCHEEGANS (1928-1934), et par HAAS \& EMELIANOFF (1933-1936): la synthèse de cette phase de recherches est publiée par V. HOURCQ (1943) et fournit des renseignements sur la série sédimentaire en bordure du bassin et ses fenêtres (crétacé inférieur et crétacé supérieur). Au cours des années 50, la Société des Pétroles d'Afrique Equatoriale (SPAFE) reprend ses études dans la plaine côtière et décrit la stratigraphie du bassin dans divers publications. La mise en œuvre des méthodes de prospections géophysiques ont permis de :

- préciser la structure du bassin côtier congolais ;

- découvrir le gisement de pétrole et de gaz de la Pointe-Indienne (1951);

- fournir une coupe stratigraphique du bassin, à l'aide des forages profonds.

A partir de 1968, les découvertes de pétrole offshore du Gabon et du Cabinda, incitent deux sociétés (Elf-Congo et Agip-Congo) à demander les permis d'exploration au large des côtes congolaises.

C'est ainsi que furent découverts les premiers gisements offshores dans le bassin côtier congolais, qui sont :

- Emeraude : c'est le premier gisement offshore, qui est situé à $17 \mathrm{~km}$ des côtes et $30 \mathrm{~km}$ au sud de la pointe-indienne. La production de ce gisement débuta en 1972.

- Louango : découvert en 1971 au nord d'Emeraude. C'est le deuxième gisement offshore, qui débuta sa production en été 1977.

- Likouala : découvert en août 1972 à $22 \mathrm{~km}$ à l'ouest d'Emeraude, à une profondeur entre $1270 \mathrm{~m}$ et $1365 \mathrm{~m}$. sa production débuta en mars 1980 .

- Yanga et Koundji : ce sont deux autres gisements découverts en 1979, entre les gisements d'Emeraude et de Louango.

Ces cinq gisements ont donc contribués au démarrage de la croissance de production pétrolière annuelle du Congo, qui débuta timidement (en on-shore) en 1957. Cette production annuelle s'éleva donc régulièrement depuis 25 ans pour atteindre 4,6 millions de mètre cube à la fin de 1981 .

Jusqu'à la fin de 1993, les réserves prouvées du pétrole au Congo n'avaient jamais dépassées 800 millions de barils. C'est à partir de 1994 que les réserves sont passées d'environ 700 millions à 1,7 milliard de barils (entre 1980 et 1999), avant de se stabiliser autour de 1,6 milliard de barils jusqu'en 2013. Au fur et à mesure que les recherches s'opèrent, les nouvelles découvertes font augmenter les réserves.

De 1994 à 2013, le rythme annuel de croissance de la production pétrolière au Congo est en moyenne d'environ 3\% avec un pic en 2010. Plusieurs facteurs peuvent expliquer cette pression, parmi lesquels on pourra citer : 


\section{Hors session}

- L'intensité des recherches et des investissements, rendue possible grâce au volume des réserves prouvées (1,6 milliard de barils) dans le bassin côtier ;

- Le cadre juridique et fiscal notamment le code des hydrocarbures de 1994 a contribué à l'attraction des investissements pétroliers avec la vulgarisation du contrat de partage de production ;

- Le prix du baril de cette époque sur le marché pétrolier a rendu économiquement rentable l'exploitation pétrolière.

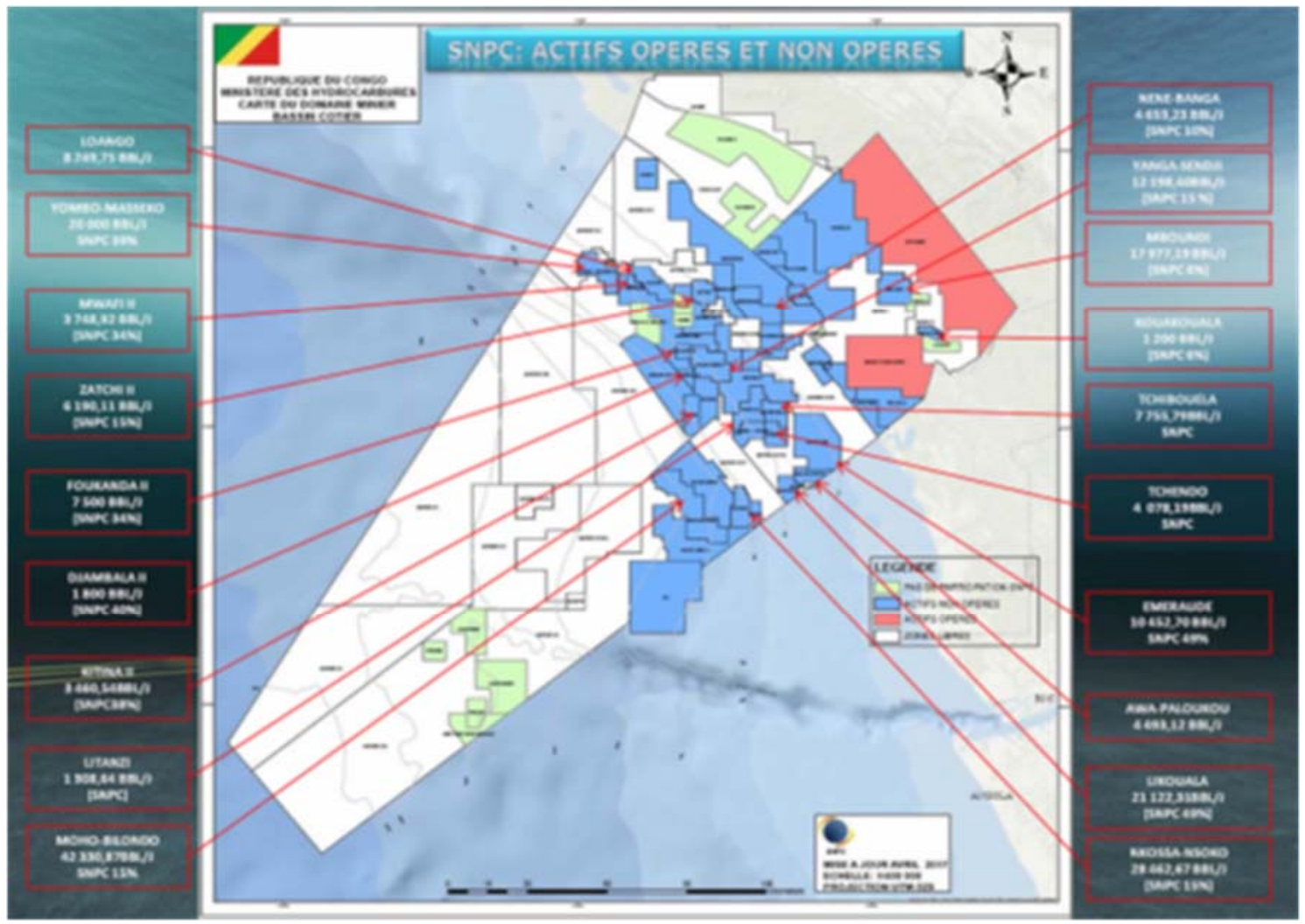

Figure 4. Production pétrolière journalière en 2017, (source SNPC, 2017c).

Les pétroles du bassin côtier Congolais sont évalués par rapport au Brent et classifiés au sein de trois catégories suivantes de brut, selon leur degré API (American Petroleum Institute) :

- Le Nkossa Brent (pétrole léger avec un degré API comprise entre $31,1^{\circ}$ et $35^{\circ} \mathrm{API}$ ) ;

- Le Djeno Brent et Azurite (pétrole moyen si 1'API est compris entre 22,3 et 31,1; soit autour de $27^{\circ}$ API pour Djeno et $25^{\circ}$ API pour Azurite) ;

- Le Yombo (pétrole lourd si l'API est compris entre $10^{\circ}$ et $22,3^{\circ}$ ).

$\mathrm{Au}$ rythme actuel de production, l'épuisement des réserves actuelles du Congo peut intervenir à partir des années 2060.

En 2017, la production journalière des gisements des hydrocarbures du bassin côtier était estimée selon la figure 4. 


\section{XVIèmes Journées Nationales Génie Côtier - Génie Civil \\ Le Havre 2020}

\subsection{Le gaz}

Le secteur gazier au Congo est encore embryonnaire malgré les importantes réserves possibles, les champs à fort potentiel en gaz ne sont pas encore exploités, l'essentiel de la production est le gaz associé au pétrole dont $61 \%$ est réinjecté et $23 \%$ est torché.

Les ressources gazières du Congo sont composées de :

- Du gaz naturel, dont la majorité des champs découverts ne sont pas encore exploités;

- Du gaz associé au pétrole.

Les réserves prouvées en gaz sont estimées à :

- Environ 268 milliards de Sm3 (mètre cube standard) en 2013, dont 182 milliards sous forme de gaz naturel brut et 86 milliards de gaz associé au pétrole ;

- 167 milliards de standards Sm3 en 2010.

Selon l'EIA (Energy Information Administration), le Congo posséderait des réserves possibles pouvant aller jusqu'à plus de 3000 milliards de Sm3 de gaz naturel (Voir figure 5).

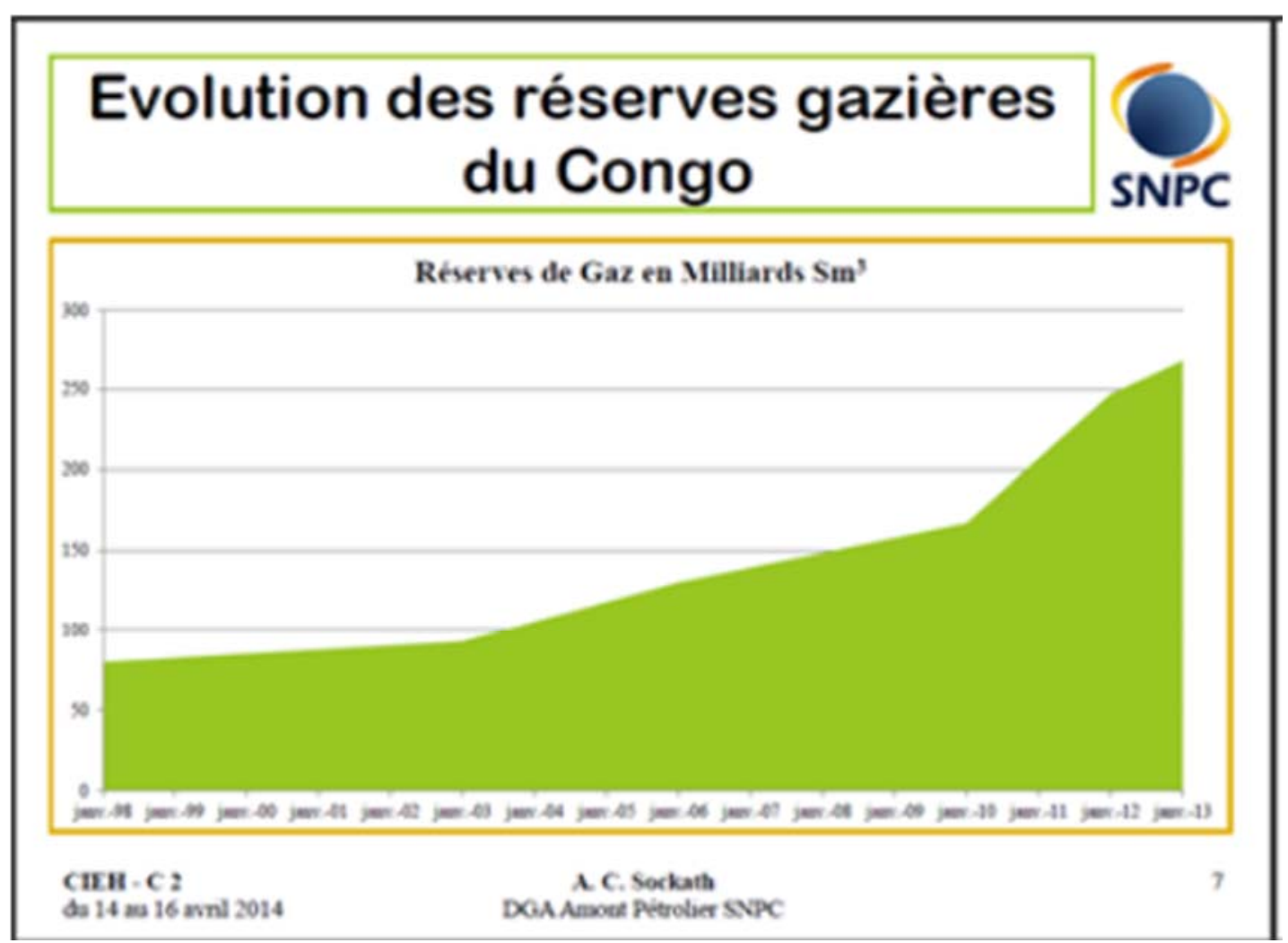

Figure 5. Réserves de gaz du bassin côtier, source (SOCKATH - SNPC, 2014).

La production du gaz associé était en forte croissance entre 1994 et 2012, elle était passée de 47 à 335,1 milliards de pieds cubes, avec une chute sensible entre 1995 et 2002. Depuis $2002,61 \%$ de gaz produit est réinjecté dans les puits, $23 \%$ est torché et seulement $16 \%$ 


\section{Hors session}

est commercialisé, valorisé, exporté, autoconsommé ou affecté pour la production de l'électricité.

En 2013, les principaux champs pétroliers en production renfermant une quantité importante de gaz étaient :

- Nkossa : avec 4,13 milliards Sm3 de réserves, dont 6\% de réserves totales ;

- Mboundi : avec 6,3 milliards Sm3 de réserves, dont 10\% de réserves totales ;

- Moho-Bilondo : avec 3,2 milliards $\mathrm{Sm} 3$ de réserves, dont 5\% de réserves totales;

- Banga-Néné : avec 33,1 milliards Sm3 de réserves, dont 50\% de réserves totales ;

- Litchendjili : avec 16,8 milliards $\mathrm{Sm} 3$ de réserves, dont 26\% de réserves totales ;

- Et les autres : avec 2,16 milliards $\mathrm{Sm} 3$ de réserves, dont 3\% de réserves totales.

Le champ de Banga-Néné détenait alors la moitié de l'essentiel des réserves en gaz associé du bassin côtier congolais (voir figure 6).

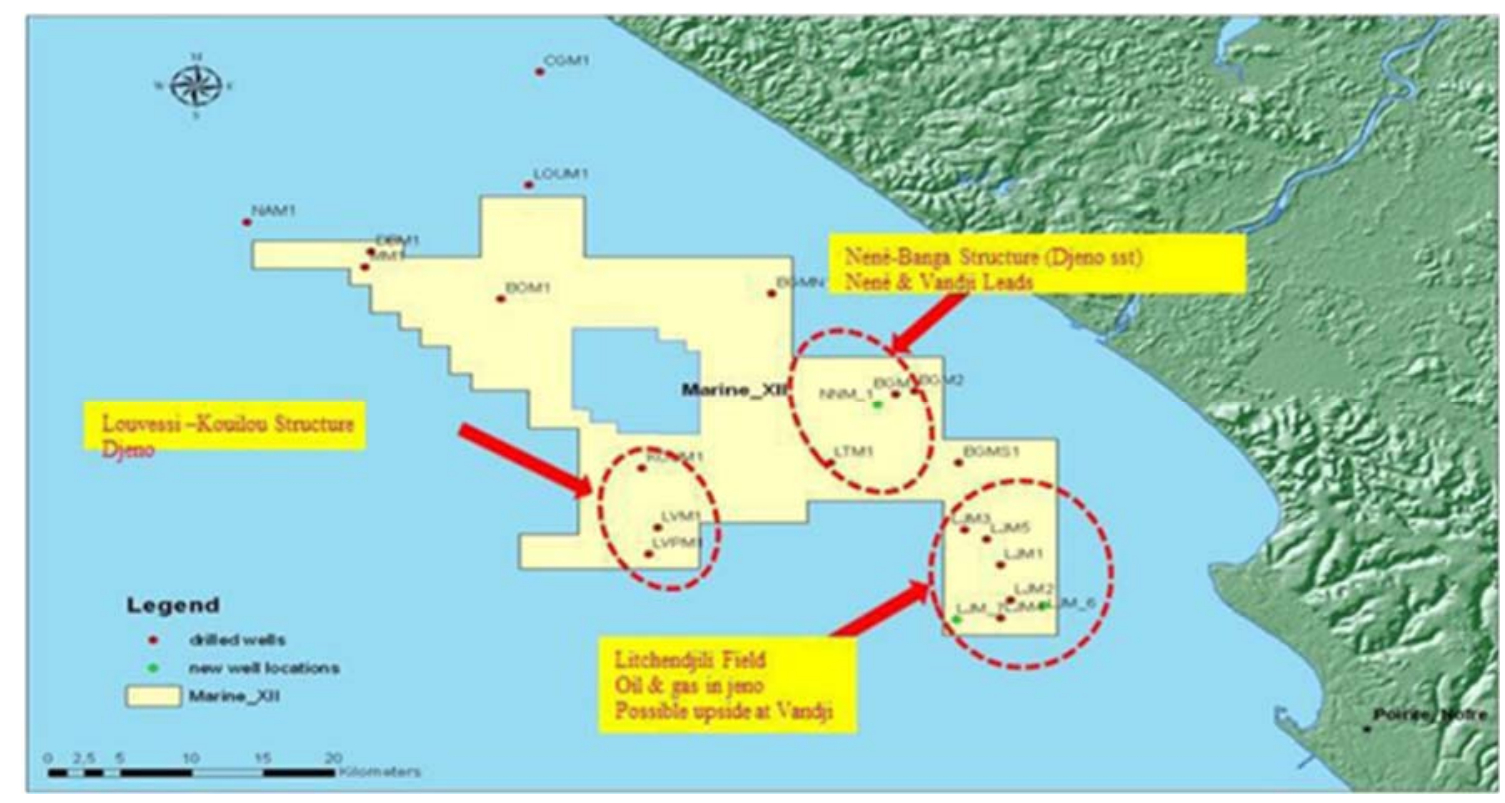

Figure 6. Région à forte concentration des réserves en gaz associé de l'offshore du bassin côtier congolais (SOCKATH - SNPC, 2014).

\subsection{Les zones libres}

Plusieurs champs pétroliers en production sont actuellement en phase mature dans le bassin côtier, d'où la nécessité de promouvoir les zones libres pour relancer la production. C'est ainsi que nous allons prendre en revue quelques champs de ces zones libres déjà explorées :

La zone $X X V$ voir figure 7, dont les résultats des forages de prospections avec succès, montrent que :

- En 1973 : * KAM-1 à une profondeur de 1652m, découvre des indices de pétrole (AGIP-CONGO) ; *KIM-1 à une profondeur de 2223m; découvre des indices de pétrole (AGIP-CONGO). 


\section{XVİ̀mes Journées Nationales Génie Côtier - Génie Civil \\ Le Havre 2020}

- En 1974 : *LEM-1 à une profondeur de 1727m, découvre du pétrole (ELF-CONGO).

- En 1980: *MASM-1 à une profondeur de 2302m, découvre du gaz naturel (AGIPCONGO).

- En 1982: *LMS-1x à une profondeur de 4460m, découvre du pétrole (CITIES SERVICES).

- En 1985 : *GOM-2 à une profondeur de 4341,5m, découvre du gaz naturel (AMOCO).

- En 1988 : *SKM-1 à une profondeur de 2133m, découvre du gaz naturel (AMOCO) ; *MBM-1 à une profondeur de 1800m, découvre du pétrole (AMOCO).

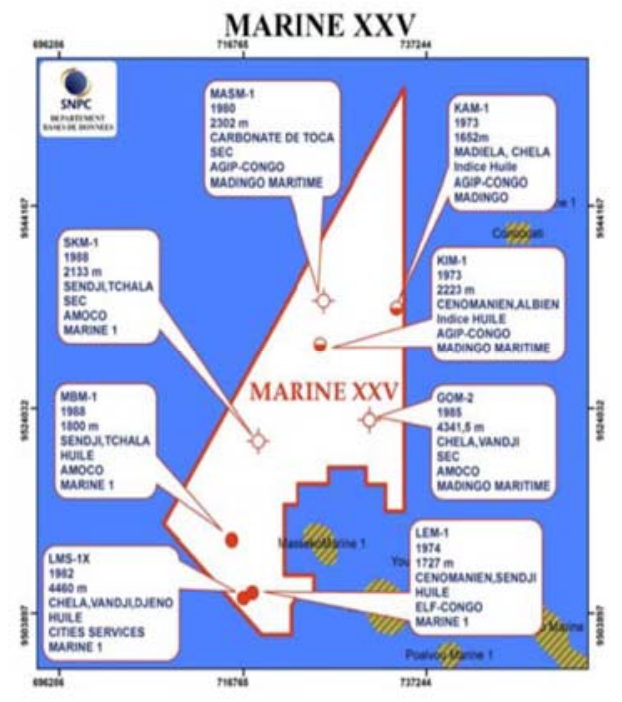

Figure 7. La zone marine $X X V$.

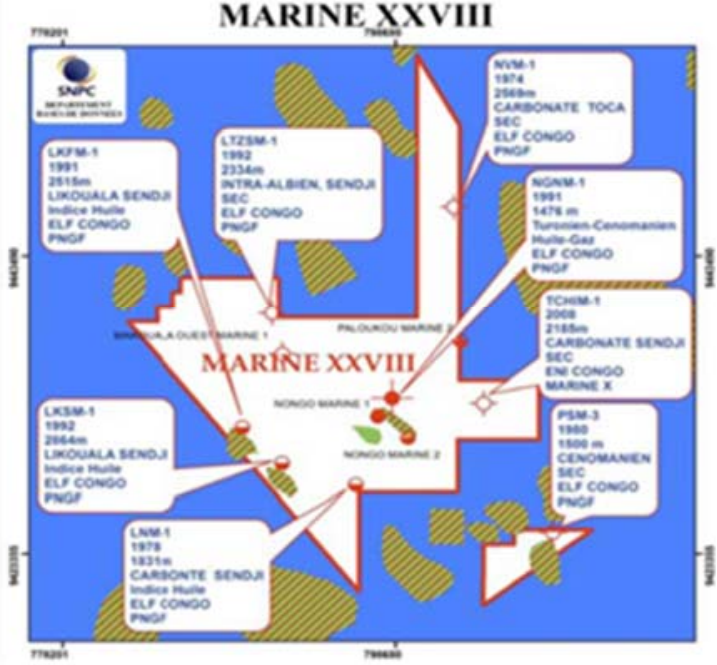

Figure 8. La zone marine XXVIII. (figures 7 et 8, source SNPC, 2017d).

La zone $X X V I I I$ voir figure 8 , dont les forages de prospections avec succès, nous montre que :

- En 1974 : *MVM-1 à 2569m de profondeur, découvre du gaz naturel (ELF-CONGO).

- En 1978 : *LNM-1 à $1831 \mathrm{~m}$ de profondeur, découvre des indices de pétrole (ELFCONGO).

- En 1980 : *PSM-3 à 1500m de profondeur, découvre du gaz (ELF-CONGO).

- En 1991 : *LKFM-1 à 2515m de profondeur, découvre les indices de pétrole (ELFCONGO) ; *NGNM-1 à 1476m de profondeur, découvre du pétrole associé au gaz (ELF-CONGO).

- En 1992: *LTZSM-1 à 2334m de profondeur, découvre du gaz (ELF-CONGO); *LKSM-1 à $2864 \mathrm{~m}$ de profondeur, découvre les indices de pétrole (ELF-CONGO).

- En 2008 : *TCHIM-1 à 2185m de profondeur, découvre du gaz (ENI-CONGO).

La zone MARINE XXIX, voir figure 9, dont quatre forages de prospection ont été réalisées, montre que : 


\section{Hors session}

- En 1970 : *DM-1, à 4062m de profondeur, découvre des indices du gaz associé au pétrole (AGIP-CONGO).

- En 1989 : *KGM-1, à 2650m de profondeur, découvre du pétrole (CONOCO).

- En 1992: *NGOM-1, à 2550m de profondeur, découvre des indices de pétrole (CONOCO).

- En 2009 : *LYM-1, à 1140m de profondeur, découvre du gaz (SOCO).

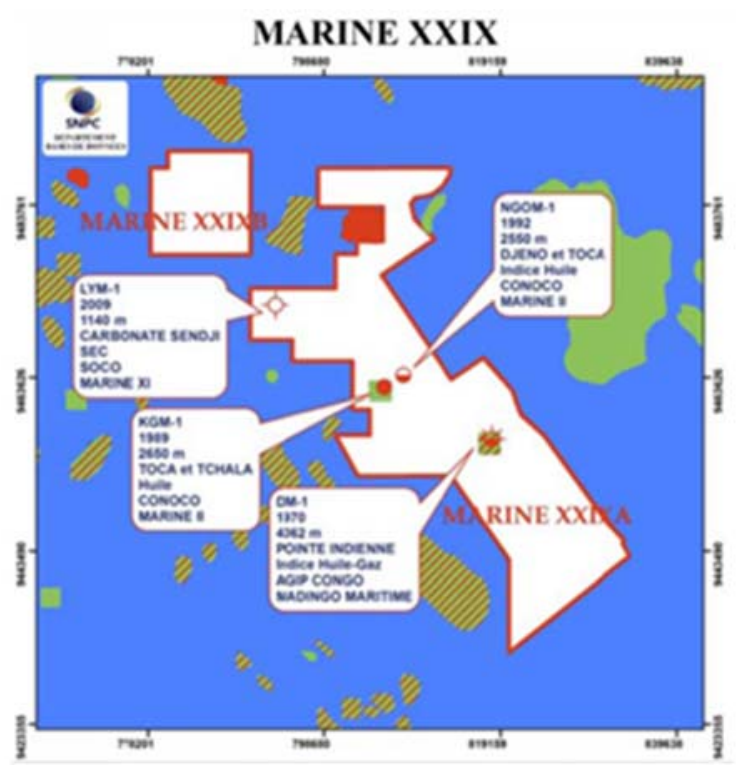

Figure 9. La zone marine XXIX.

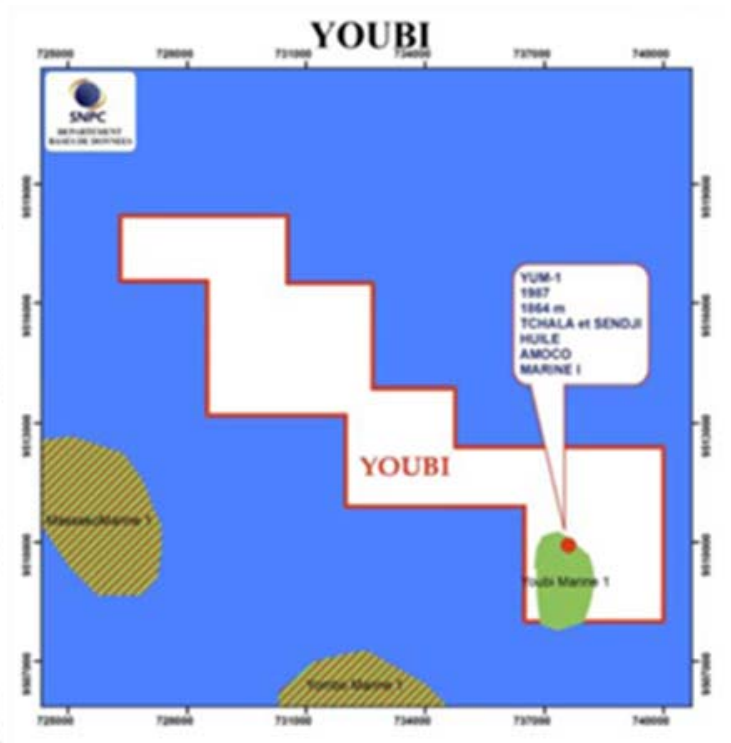

Figure 10. La zone marine Youbi.

(figures 9 et 10, source SNPC, 2017d).

La zone YOUBI, voir figure 10, dont un forage de prospection a été réalisé avec succès, montre que :

- En 1987 : *YUM-1, à 1864m de profondeur, découvre du pétrole (AMOCO).

- La zone XXVI, voir figure 11, dont dix forages de prospections ont été réalisés avec succès, et montrent que :

- En 1963 : *CMG-1, à 2254,3m de profondeur découvre des indices de pétrole (ELFCONGO)

- En 1972 : *VDM-1, à 3389m de profondeur, découvre des indices de pétrole (ELFCONGO) ; *KOM-1, à 1067m de profondeur, découvre du gaz (AGIP-CONGO).

- En 1974: *NAM-1, à 1578m de profondeur, découvre du gaz (AGIP-CONGO) ; *COM-1, à $1962 \mathrm{~m}$ de profondeur, découvre du gaz associé au pétrole (AGIPCONGO).

- En 1979 : *MIKM-1, à 1714m de profondeur, découvre du pétrole (AGIP-CONGO).

- En 1981 : *PALM-1, à 1356m de profondeur, découvre du gaz (AGIP-CONGO).

- En 1984 : *BEM-1, à 2719m de profondeur, découvre du pétrole (ELF-CONGO).

- En 1986 : *BUGM-1, à 2743m de profondeur, découvre du gaz (AGIP-CONGO). 


\section{XVİ̀mes Journées Nationales Génie Côtier - Génie Civil \\ Le Havre 2020}

- En 1987 : *LOUM-1, à 2206m de profondeur, découvre du gaz (AMOCO).

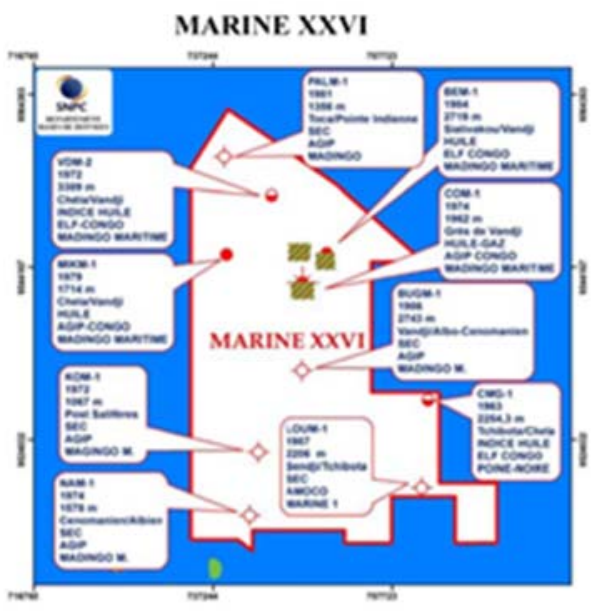

Figure 11. La zone marine XXVI.

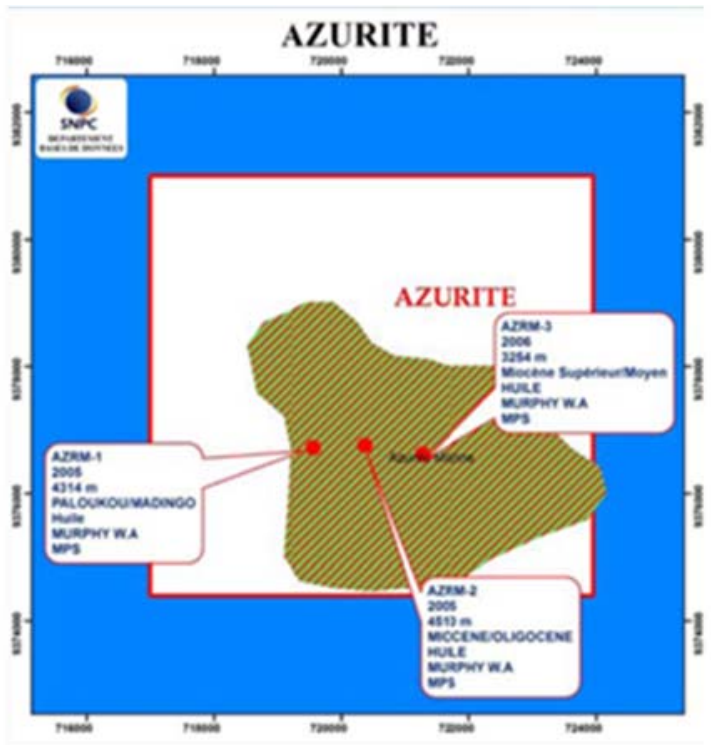

Figure 12. La zone marine Azurite.

(figures 11 et 12, source SNPC, 2017d).

La zone AZURITE, voir figure 12, dont trois forages de prospections ont été réalisés, et montrent que :

- En 2005 : *AZRM-1, à 4314m de profondeur, découvre du pétrole (MURPHY W.A) ; *AZRM-2, à 4510m de profondeur, découvre du pétrole (MURPHY W.A).

- En 2006 : *AZRM-3, à 3254m de profondeur, découvre du pétrole (MURPHY W.A).

- La zone XXXI, voir figure 14, dont quatre forages de prospections ont été réalisés, et montrent que :

- En 1970 : *MM-1, à 2762m de profondeur, découvre du pétrole (AGIP-CONGO).

- En 1973 : *BOM-1, à 2187m de profondeur, découvre du gaz (AGIP-CONGO).

- En 1982 : *KOUM-1, à 3519m de profondeur, découvre du pétrole (AGIP-CONGO).

- En 1992 : *DBM-1, à 1603m de profondeur, découvre du gaz (ARCO).

La zone XXXII, voir figure 13, dont quatre forages de prospections ont été réalisés avec succès, et montrent que :

- En 2009 : *DMNM-1, à 3996m de profondeur, découvre du gaz (MURPHY W.A).

- En 2010 : *TRQM-1, à 3677m de profondeur, découvre du pétrole (MURPHY W.A) ; *TRQM-3, à 3301m de profondeur, découvre du gaz (MURPHY W.A) ; *TRQM-4, à $4568 \mathrm{~m}$ de profondeur, découvre du pétrole (MURPHY W.A). 


\section{Hors session}

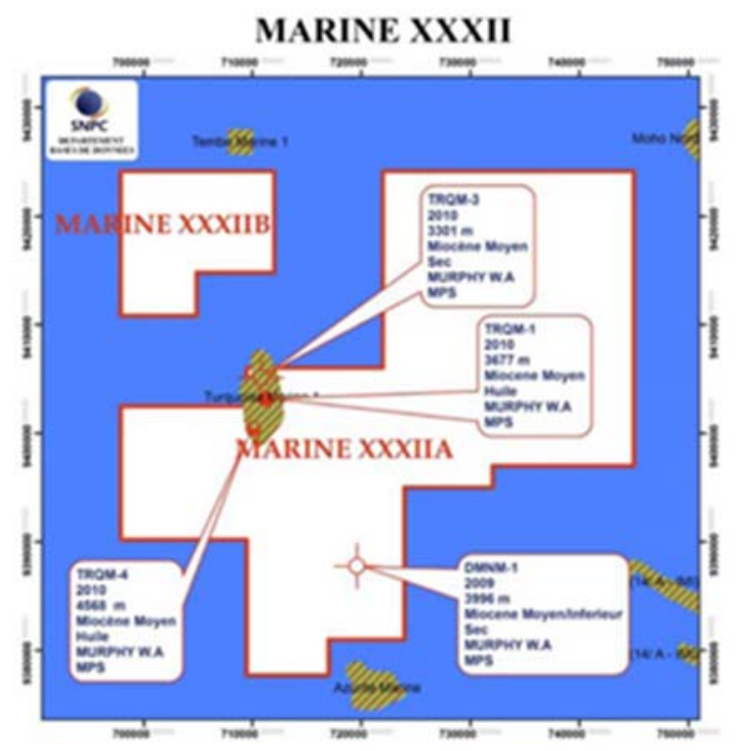

Figure 13. La zone marine XXXII.

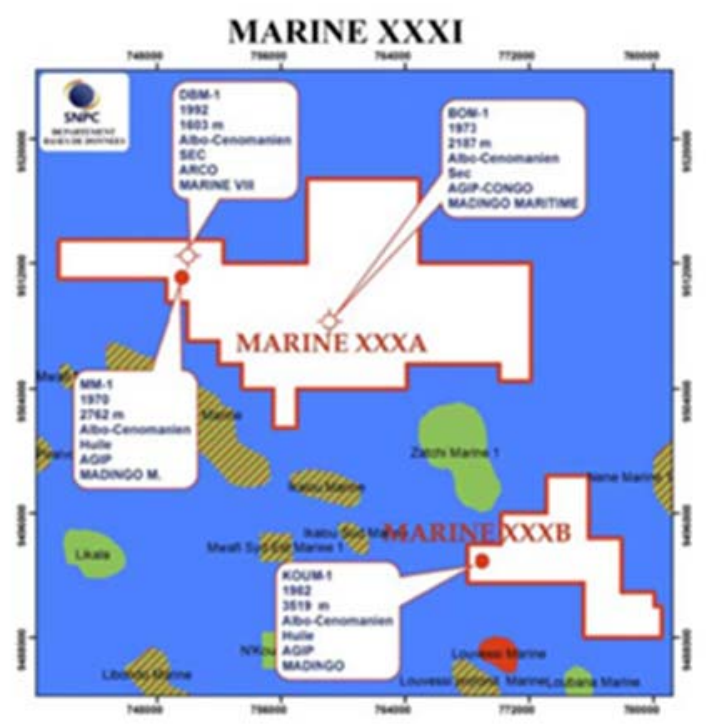

Figure 14. La zone marine XXXI.

(figures 13 et 14, source SNPC, 2017d).

\section{Conclusion}

Pour préparer l'avenir, les sociétés pétrolières opérantes au Congo, en particulier TOTAL E\&P CONGO (2017) se sont donné l'obligation de découvrir de nouveaux gisements et de renouveler les réserves des hydrocarbures. C'est ainsi qu'une exploration «near-by » est menée sur le bassin, et a pour objectifs principaux d'identifier, d'évaluer et d'explorer les nouvelles structures ou d'apprécier les structures connues à proximité ou sur les champs existants. Un effort sera aussi porté pour une exploration avancée sur les formations sous-explorées, souvent enfouies comme les réservoirs « anté-salifères ».

\section{Bibliographie}

HOURCQ V. (1943). Esquisse de la géologie de l'A.E.F., les terrains sédimentaires de la région côtière, Bull. Serv. Mines A.E.F., ${ }^{\circ} 1$, Le Caire.

SNPC (2017a). Carte du bassin côtier, Société Nationale des Pétroles du Congo.

SNPC (2017b). Congo mega survey, Société Nationale des Pétroles du Congo.

SNPC (2017c). Actifs opérés et non opérés SNPC, Société Nationale des Pétroles du Congo.

SNPC (2017d). Zones libres, Société Nationale des Pétroles du Congo.

SOCKATH A. C. - SNPC (2014). Potentiel et valorisation des ressources gazières, $2^{\text {ième }}$ Conférence Internationale et Exposition sur les Hydrocarbures au Congo, Avril 2014, Brazzaville, Congo.

TOTAL E\&P (2017). Exploration : préparer l'avenir, Documentation. www.cg.total.com 